\title{
Microscopic and Instrumental Analysis of Particulate Matter related to Parental Products and Devices
}

\author{
K. Ashline, S. Strathmann
}

Baxter Technology Resources, Route 120 and Wilson Road, Round Lake, Illinois 60073

Particulate matter analysis differs from the traditional trace analytical samples in that particulate matter by definition is heterogeneously dispersed in various matrices and requires isolation prior to analysis. The path of analysis most frequently applied in particle identification represents a stepwise progression from low to high magnification and from simple to complex techniques [1]. Compositional analysis of particles isolated from pharmaceutical and parenteral products is accomplished through the use of micro-techniques; microscopy, energy dispersive x-ray spectroscopy (EDXS), micro-Fourier transform infrared (FT-IR), and more recently micro-Raman spectroscopy. Less frequently, mass spectrometry or diffraction techniques are applied. Stereomicroscopy and polarized light microscopy provide information on morphology and constitutes a means of selecting target particles for micro-chemical analyses. The EDXS gives elemental composition and FT-IR and Raman spectroscopy provides molecular information. Using these techniques it is possible to characterize, and in most cases, identify, the particulate matter of interest.

Historically, our laboratory has used EDXS as a method to determine the elemental make-up of single particles. However, EDXS maps provide both elemental information and spatial information on the elements within a sample. The x-ray map for a cross-section of "bubble" switch is shown in Figure 1. The map shows the location of aluminum, silicon, carbon, titanium, chlorine, oxygen, and magnesium. Overlapping elements in the same location can indicate the presence of a particular compound, for example magnesium silicate, at the surface of the bubble switch.

Micro-FTIR is another technique which has traditionally been applied to the analysis of individual particles. However, with the use of a focal-plane array detector microscopic spatial imaging can be used to generate spectroscopic chemical images. The photomicrograph and FT-IR image of a calcium stearate crystal on a polyethylene film are shown in Figures 2 and 3. A FT-IR spectrum of a point on the calcium stearate crystal is also shown in Figure 4. The array detector enables full range spectra from all the pixels to be collected simultaneously yielding 4096 spectra for a 64 x 64 detector.

A challenge in particle analysis is to demonstrate that particles with the same morphology and different sizes are the same chemically. Micro-Raman spectrometry is a complementary technique to micro FT-IR and it has the advantage of being able to probe particles of a much smaller size. The photomicrograph and micro FT-IR spectrum of a spherical particle approximately $100 \mu \mathrm{m}$ in size is shown in Figure 5. The scanning electron photomicrograph and micro Raman spectrum of an approximately $7 \mu \mathrm{m}$ spherical particle are shown in Figures 7 and 8. The FT-IR and Raman spectra confirm the composition of both particles is the same.

[1] D. S. Aldrich and M. A. Smith, Practical Guide to Infrared Microspectroscopy, 1995, Chapter 9. 

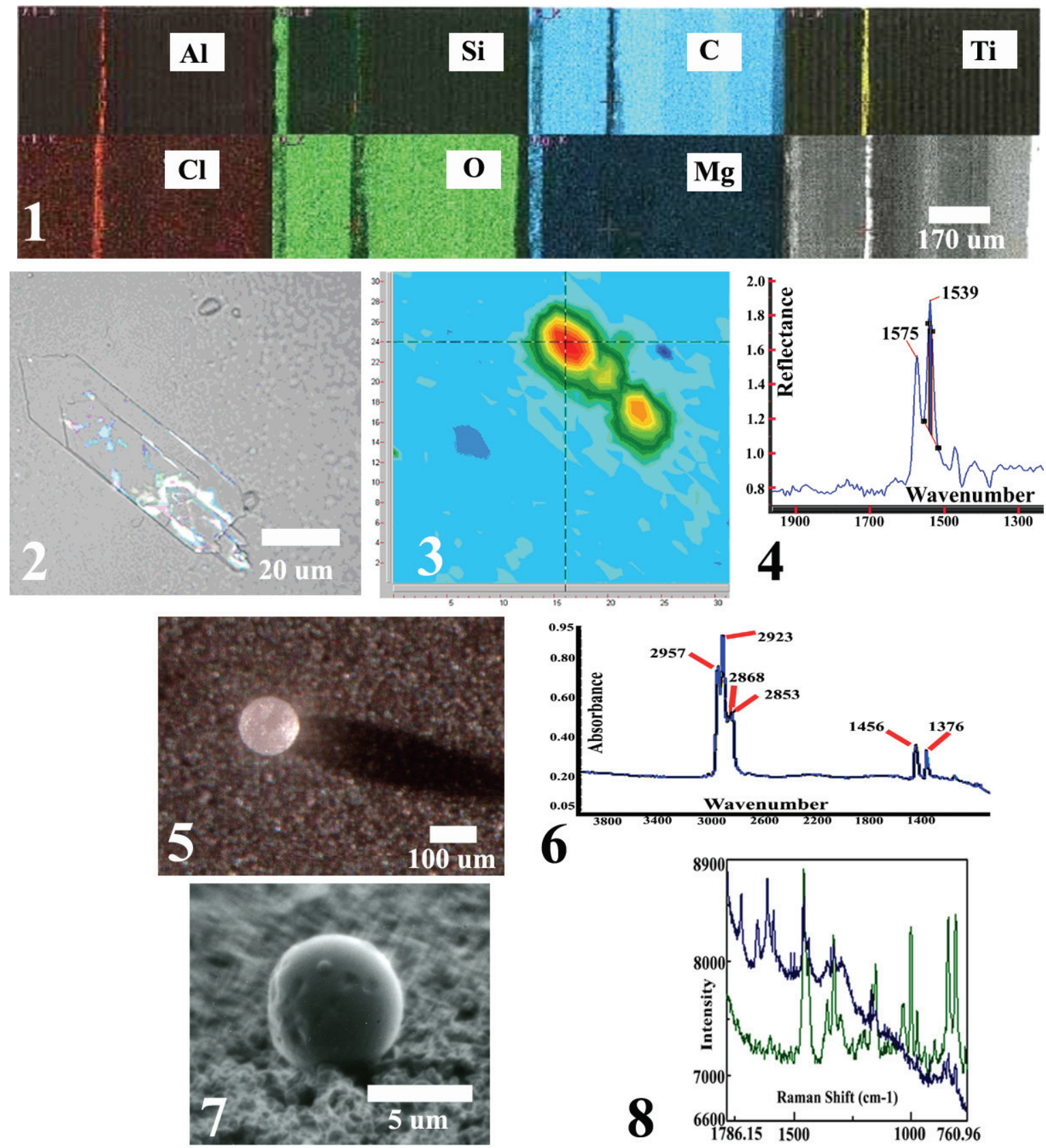

Figure 1. X-ray map of a cross-section of plastic film used to manufacture a bubble switch. Figure 2. Photomicrograph of a calcium stearate crystal on a polyethylene film.

Figure 3. The FT-IR chemical image of the calcium stearate crystal (Figure 2). on polyethylene film. Figure 4. Infrared spectrum of the crystal (Figures 2) at the location indicated by the cross hairs in Figure 3.

Figures 5, 6. Photomicrograph of $129 \mu \mathrm{m}$ sphere and its micro-FT-IR spectrum.

Figure 7. Scanning electron photomicrograph of a $6 \mu \mathrm{m}$ sphere. The sphere in Figures 6 and 7 were obtained from the same particle population.

Figure 8. Micro-Raman spectrum of the $6 \mu \mathrm{m}$ sphere (upper) overlaid on the Raman spectrum of polypropylene. 